\title{
Exploring the impact of efficacy messages on cessation- related outcomes using Ecological Momentary Assessment
}

\author{
Victoria Lambert', Stuart G. Ferguson², Jeff Niederdeppe ${ }^{3}$, David Hammond ${ }^{4}$, James W. Hardin' ${ }^{5}$, James F. Thrasher ${ }^{1,6}$
}

\begin{abstract}
INTRODUCTION Observational studies indicate that cigarette package inserts with efficacy messages about the benefits of quitting (i.e. response efficacy) and recommendations for successful cessation increase smokers' self-efficacy to quit and promote sustained cessation. However, the effects of inserts with such efficacy messages have not been studied using experimental designs. This study used ecological momentary assessment (EMA) to assess smokers' responses to efficacy inserts.

METHODS In a randomized case-crossover study among smokers from the United States $(n=23)$, participants received a one-week supply of cigarettes with efficacy inserts and a one-week supply without any inserts, and were randomized to use the packs with inserts on either the first or second week of the study. For 14 consecutive days, participants used a smartphone to answer brief surveys on cessation-related variables during smoking sessions and at the beginning of each day. Multilevel mixed-effects linear and logistic regression models compared responses during the insert period to those of the non-insert period.

RESULTS The insert period was associated with greater desire to quit ( $b=0.21$, $\mathrm{p}=0.012)$, motivation to quit $(\mathrm{b}=0.18, \mathrm{p}=0.001)$, self-efficacy to cut down $(b=0.26, p<0.001)$ and to quit $(b=0.28, p<0.000)$, and response efficacy/ perceived benefits of quitting $(b=0.13, p=0.012)$. Insert exposure was not significantly associated with forgoing cigarettes $(\mathrm{OR}=1.9, \mathrm{p}=0.2)$.

CONCLUSIONS Results from this EMA study suggest that inserts with efficacy messages may promote determinants of smoking cessation. This is consistent with observational research in Canada, which is the only country to use inserts with efficacy messages as well as pictorial warnings about smoking risks on the outside of packs. Future studies should assess the extent to which efficacy inserts can not only be used to communicate health information to smokers but also work in synergy with pictorial warnings.
\end{abstract}

\author{
AFFILIATION \\ 1 Department of Health \\ Promotion, Education, \& \\ Behavior, Arnold School of \\ Public Health, University of \\ South Carolina, Columbia, \\ United States \\ 2 School of Medicine, \\ University of Tasmania, \\ Hobart, Australia \\ 3 Department of \\ Communication, Cornell \\ University, Ithaca, United \\ States \\ 4 School of Public Health \&t \\ Health Systems, University of \\ Waterloo, Waterloo, Canada \\ 5 Department of \\ Epidemiology \& Biostatistics, \\ Arnold School of Public \\ Health, University of South \\ Carolina, Columbia, United \\ States \\ 6 Department of Tobacco \\ Research, Center for \\ Population Health Studies, \\ National Institute of Public \\ Health, Cuernavaca, Mexico \\ CORRESPONDENCE TO \\ Victoria Lambert. Department \\ of Health Promotion, \\ Education, \& Behavior, Arnold \\ School of Public Health, \\ University of South Carolina, \\ 915 Greene Street, 29201 \\ Columbia, United States. \\ E-mail: vlambert@email. \\ sc.edu \\ KEYWORDS \\ smoking cessation, health \\ policy, tobacco control, health \\ communication
}

Received: 5 June 2018 Revised: 17 August 2018

Accepted: 20 August 2018

\section{INTRODUCTION}

While many countries and jurisdictions have implemented pictorial health warning labels
(HWLs) on the outside of cigarette packs, Canada is the only country that also requires complementary messaging on inserts inside the packs. Canadian 
inserts include supportive health messages on the benefits of quitting (i.e. response efficacy messages) and recommendations for successful smoking cessation (i.e. self-efficacy messages). Observational studies suggest that these inserts increase smokers' self-efficacy to quit ${ }^{1}$ and promote quit attempts and sustained smoking cessation ${ }^{1,2}$. Further, experimental studies indicate that smokers perceive inserts with Canadian-style efficacy messages to be motivating and useful for quitting.3,4. The current study assessed the real-world effects of inserts on cessation-related variables among a sample of smokers from the United States.

The cessation-related effects of pictorial HWLs that highlight smoking risks using graphic imagery appear to be partially due to increased negative affect, including fear ${ }^{5}$. As suggested by fear-appeal research and theories ${ }^{6,7}$, a combination of self-efficacy and response efficacy messages can enhance fearappeal messages and promote desirable attitudinal and behavioral change independent of fear arousal. However, the effects of inserts with efficacy messages have not been assessed in experiments outside brief, one-time exposures to messages online ${ }^{3,4}$. Further research is needed to determine if these supportive messages are effective.

This study used ecological momentary assessment (EMA) methods to assess smokers' real-time responses to inserts with efficacy messages in the US, where warning labels are small and do not include pictures. EMA has been used in observational studies to assess smokers' responses to pictorial HWLs with fear-arousing content ${ }^{8-10}$, but not to assess responses to efficacy messages on inserts. While the primary purpose of this study was to test the feasibility of the study procedures, we expected that the period of exposure to efficacy inserts would be associated with greater self-efficacy to reduce and completely quit smoking, response efficacy, motivation and desire to quit, and forgoing cigarettes, when compared to the period without exposure to efficacy inserts.

\section{METHODS}

\section{Sample}

Data collection occurred between 19 July 2017 and 11 February 2018, in South Carolina and New York. Smokers in South Carolina were recruited through e-mail, Facebook, Craigslist, and Instagram advertisements. Smokers in New York were recruited through word-of-mouth and street intercepts outside tobacco shops. Potential participants completed an online survey to assess eligibility, which included: smoking at least 100 cigarettes in their lifetime; being a daily smoker; smoking 10 or more cigarettes per day; being 18-50 years of age; planning to quit sometime in the future; and no use of e-cigarettes or any other combustible/smokeless tobacco product in the prior month.

\section{Study protocol}

EMA involves using mobile devices to query participants at critical moments, such as during smoking sessions when people are exposed to messages on and in cigarette packages, potentially mitigating recall bias ${ }^{11}$. For this study, EMA data were collected using smartphones programmed with a customized app installed and other device functions disabled (http://www.utas.edu.au/health/research/ groups/behavioural-and-situational-research-groupbsrg/hbart).

Participants attended a pre-study orientation that involved: confirmation of smoking status using a carbon monoxide breathalyzer (minimum 8 ppm CO required); a brief survey; and training in the study protocol. Participants were given a two-week supply of their preferred cigarette brand variety: one week's supply with inserts and one week's supply without inserts. Participants were randomized to use the supply with inserts on either the first or second week of the study. Packs with inserts contained one of four different insert messages, and participants received at least one of each of the four messages in their supply. Of the four insert messages, two contained response efficacy information and two contained self-efficacy information, all of which included the phone number for a smoking cessation quit-line and website (smokefree.gov; see Supplementary Appendix). Each week's supply was labeled as 'Set 1 ' or 'Set 2' and placed in separate, zip-locked bags labeled with the days on which participants should use the packs.

Participants used the smartphone provided to complete a survey each morning and to indicate each time they smoked a cigarette during the 14 consecutive days of the study period. The morning survey queried how long ago the participant had 
awaken and if they had stubbed out a cigarette early or not smoked when they intended to do so in the prior 24 hours (i.e. forgoing). As a manipulation check of insert exposure, the morning log also prompted participants to take a photo of their cigarette pack.

Participants could log cigarettes at any time of day, and, when they logged a cigarette, the app queried whether they were smoking and if the current cigarette was from a new pack. Participants were prompted to complete a brief survey (i.e. event-related survey) every time they opened a new pack and, on average, at three additional, randomly selected smoking events each day (the algorithm considered the baseline quantity of cigarettes smoked per day in determining the frequency of surveys). Event-related surveys queried feelings about smoking, desire to quit smoking, motivation to quit smoking, self-efficacy to reduce smoking, self-efficacy to quit smoking, response efficacy (i.e. perceived benefits of quitting), and worry about the effects of smoking (see Table 1 for question wording). After the 14-day EMA period ended, participants attended a de-briefing session, during which they completed an online survey on perceptions of the insert messages, participated in a qualitative interview to discuss any issues completing the study protocol, and received US\$150 in remuneration, along with a pamphlet containing information on free smoking-cessation resources.

\section{Analyses}

Multilevel mixed-effects linear and logistic models regressed outcomes from event-related surveys and morning reports on an indicator variable for the period when participants were exposed versus not exposed to inserts, controlling for week of insert exposure (i.e. 1 st vs 2 nd week). For outcomes that were measured multiple times per day (i.e. eventrelated surveys), three-level models were estimated, considering observations as nested within days

Table 1. Results for key outcomes, comparing the period with exposure versus without exposure to inserts with efficacy messages ${ }^{a}$

\begin{tabular}{|c|c|c|c|c|}
\hline Variable & Item & $\begin{array}{l}\text { Response format and } \\
\text { options (slider) }\end{array}$ & Coef: & (SE) \\
\hline Feeling toward smoking & Right now, you feel like smoking is...? & $\begin{array}{l}1 \text { = very bad } \\
\text { to } \\
7 \text { = very good }\end{array}$ & -0.10 & 0.06 \\
\hline Desire to quit & How strong is your desire to quit at this time? & $\begin{array}{l}1=\text { not at all strong } \\
\text { to } \\
7=\text { extremely strong }\end{array}$ & $0.21^{*}$ & 0.08 \\
\hline Motivation to quit & How motivated are you to quit smoking? & $\begin{array}{l}1=\text { not at all } \\
\text { to } \\
7=\text { extremely }\end{array}$ & $0.18^{* * *}$ & 0.06 \\
\hline Self-efficacy to cut down & $\begin{array}{l}\text { How easy would it be to cut down on the number of } \\
\text { cigarettes you smoke? }\end{array}$ & $\begin{array}{l}1=\text { not at all easy } \\
\text { to } \\
9=\text { extremely easy }\end{array}$ & $0.26^{* * *}$ & 0.08 \\
\hline Self-efficacy to quit & $\begin{array}{l}\text { How confident are you that you could quit smoking } \\
\text { altogether right now? }\end{array}$ & $\begin{array}{l}1=\text { not at all confident } \\
\text { to } \\
7=\text { extremely confident }\end{array}$ & $0.28^{* * *}$ & 0.06 \\
\hline Response efficacy & $\begin{array}{l}\text { How much would quitting smoking now reduce your } \\
\text { chances of getting a serious disease? }\end{array}$ & $\begin{array}{l}1 \text { = no chance } \\
\text { to } \\
7 \text { = certain to happen }\end{array}$ & $0.13^{*}$ & 0.05 \\
\hline $\begin{array}{l}\text { Worry about the risks of } \\
\text { smoking }\end{array}$ & $\begin{array}{l}\text { How worried are you about the possible effects of } \\
\text { smoking? }\end{array}$ & $\begin{array}{l}1=\text { not at all worried } \\
\text { to } \\
7=\text { extremely worried }\end{array}$ & -0.07 & 0.07 \\
\hline $\begin{array}{l}\text { Forgoing cigarettes (odds } \\
\text { ratio) }^{\mathrm{b}}\end{array}$ & $\begin{array}{l}\text { In the last } 24 \text { hours, have you stubbed out a cigarette early } \\
\text { or not had a cigarette when you would normally? }\end{array}$ & Yes/No & 1.9 & 0.90 \\
\hline
\end{tabular}


and within participants. For the forgoing outcome, which was measured once daily, two-level models considered observations nested within participants only. Stata 15 was used for all analyses (StataCorp. 2017. Stata Statistical Software: Release 15. College Station, TX: StataCorp LLC).

\section{RESULTS}

\section{Compliance and descriptive findings}

Participants reported no serious issues with completing the protocol. Of the 28 participants who began the study, 27 completed it. Four of these 27 participants were excluded from analysis because information acquired from the follow-up interview and the manipulation checks indicated they were not exposed to the inserts as assigned. Three participants did not use the insert and non-insert packs as assigned, and one participant indicated that he never saw the inserts, which was likely because they were hidden due to the type of pack for his preferred brand.

For the analytic sample of 23 smokers, the mean age was 36.2 years, and about half (52\%) was female, half intended to quit within the next 6 months (48\%), and a third (35\%) had attempted to quit in the prior 4 months. The mean Heaviness of Smoking Index ${ }^{12}$ was $3.41(\mathrm{SE}=0.22)$. The total number of cigarettes logged was 2744 (with a mean, $\mathrm{M}=8.86$ per day during insert period, and $\mathrm{M}=9.08$ per day during non-insert period). On average, participants completed 13.7 (of 14) days of the study protocol, 40.6 event-related surveys, and 10.4 (of 14) morning reports throughout the study period. Compliance with the event-related surveys ranged from 88 to $100 \%$. The average number of days exposed to inserts was 6.8 ( of 7).

\section{Insert vs non-insert period}

Table 1 shows the results from the mixed-effects regression models, comparing observations during the period when participants were exposed to inserts and when they were not. The insert period was associated with greater desire to quit $(b=0.21$, $\mathrm{SE}=0.08, \mathrm{p}=0.01)$, stronger motivation to quit $(b=0.18, S E=0.06, p=0.001)$, stronger self-efficacy to cut down $(\mathrm{b}=0.26, \mathrm{SE}=0.08, \mathrm{p}=0.001)$ and to quit $(b=0.28, S E=0.06, p<0.000)$, and greater response efficacy $(b=0.13, \mathrm{SE}=0.05, \mathrm{p}=0.01)$. Insert exposure was not significantly associated with feeling toward smoking $(b=-0.10 \mathrm{SE}=0.06, p=0.10)$, worry about the effects of smoking $(b=-0.07, \mathrm{SE}=0.07, \mathrm{p}=0.33)$ or forgoing cigarettes $(\mathrm{OR}=1.9, \mathrm{SE}=0.9, \mathrm{p}=0.2)$. The order of insert exposure (i.e. 1st vs 2 nd week) was not significantly associated with any outcome (results not shown).

\section{DISCUSSION}

The EMA protocol used in this study appears acceptable and feasible. While the findings should be confirmed in a larger study, five of the six study hypotheses regarding the positive effects of insert exposure on determinants of smoking cessation were supported. The findings that insert exposure was positively associated with self-efficacy to cut down and quit are particularly important since there is ample evidence that increasing self-efficacy promotes smoking cessation ${ }^{13-16}$.

Contrary to our hypothesized expectation, we did not find a statistically significant association between insert exposure and forgoing cigarettes, although the coefficient suggested a tendency towards more forgoing when exposed to inserts. We had relatively low power to detect this effect because data on forgoing were only collected once a day compared to multiple times a day for the other study outcomes. Larger sample sizes and longer follow-up are likely necessary to better assess forgoing cigarettes, which consistently predicts quit attempts ${ }^{17-19}$. Overall, our results suggest that inserts have medium-sized effects ${ }^{20}$ on determinants of cessation; however, these effects may partly reflect demand-effects from our case-crossover design, which did not blind participants to the experimental period. Betweensubject study designs would better control for this potential bias but would likely require larger sample sizes.

Our study had some additional limitations, including the inability to assess cessation behavior over a short period of time. Similarly, it is also unclear how long insert effects would be sustained given evidence ${ }^{21,22}$ of 'wear out' for HWLs; nevertheless, observational research in Canada found that attention to inserts increased over time, even while attention to HWLs dissipated ${ }^{1}$. More research is needed to confirm study findings and better inform cigarette labeling policy. Future 
studies should assess potentially synergistic effects of inserts and HWLs, since theory suggests that efficacy messages can enhance the effects of fear-arousing messages $^{7}$ and HWL research suggests that fear arousal might promote more deliberate processing of labeling message ${ }^{23}$. Furthermore, although theory emphasizes the importance of both response efficacy and self-efficacy messages, future research could investigate this premise by assessing their independent effects on cessation-related outcomes.

\section{CONCLUSIONS}

The preliminary findings from this pilot study suggest that efficacy messages promote determinants of cessation. These findings warrant further assessment in larger trials that randomize participants to different labeling conditions, including assessment of potential synergies between efficacy messages and fear-arousing messages.

\section{REFERENCES}

1. Thrasher JF, Swayampakala K, Cummings KM, et al. Cigarette package inserts can promote efficacy beliefs and sustained smoking cessation attempts: A longitudinal assessment of an innovative policy in Canada. Prev Med. 2016;88:59-65. doi:10.1016/j.ypmed.2016.03.006

2. Thrasher JF, Osman A, Abad-Vivero EN, et al. The Use of Cigarette Package Inserts to Supplement Pictorial Health Warnings: An Evaluation of the Canadian Policy. Nicotine Tob Res. 2015;17(7):870-875. doi:10.1093/ntr/ntu246

3. Thrasher JF, Anshari D, Lambert-Jessup V, et al. Assessing Smoking Cessation Messages with a Discrete Choice Experiment. Tob Regul Sci. 2018;4(2):73-87. doi:10.18001/TRS.4.2.7

4. Thrasher JF, Islam F, Davis RE, et al. Testing cessation messages for cigarette package inserts: Findings from a best/worst discrete choice experiment. Int J Environ Res Public Health. 2018;15(2):1-21. doi:10.3390/ijerph15020282

5. Cho YJ, Thrasher JF, Yong HH, et al. Path analysis of warning label effects on negative emotions and quit attempts: A longitudinal study of smokers in Australia, Canada, Mexico, and the US. Soc Sci Med. 2018;197:226234. doi:10.1016/j.socscimed.2017.10.003

6. Tannenbaum MB, Hepler J, Zimmerman R, et al. Appealing to fear: A meta-analysis of fear appeal effectiveness and theories. Psychol Bull. 2015;141(6):1178-1204. doi:10.1037/a0039729

7. Witte K. Putting the fear back into fear appeals: The extended parallel process model. Commun Monogr. 1992;59(4):329-349.doi:10.1080/03637759209376276
8. Schüz N, Ferguson SG. Australian smokers' and nonsmokers' exposure to antismoking warnings in day-to-day life: A pilot study. Nicotine Tob Res. 2015;17(7):876-881. doi:10.1093/ntr/ntu253

9. Schüz N, Eid M, Schüz B, Ferguson SG. Immediate effects of plain packaging health warnings on quitting intention and potential mediators: Results from two ecological momentary assessment studies. Psychol Addict Behav. 2016;30(2):220-228. doi:10.1037/adb0000146

10. Mays D, Murphy SE, Johnson AC, Kraemer JD, Tercyak KP. A pilot study of research methods for determining the impact of pictorial cigarette warning labels among smokers. Tob Induc Dis. 2014;12(1). doi:10.1186/1617-9625-12-16

11. Shiffman S, Stone AA, Hufford MR. Ecological Momentary Assessment. Annu Rev Clin Psychol. 2008;4(1):1-32. doi:10.1146/annurev.clinpsy.3.022806.091415

12. Heatherton TF, Kozlowski LT, Frecker RC, Rickert W, Robinson J. Measuring the Heaviness of Smoking: using self-reported time to the first cigarette of the day and number of cigarettes smoked per day. Br J Addict. 1989;84(7):791-800. doi:10.1111/j.1360-0443.1989.tb03059.x

13. Andrews JO, Felton G, Wewers ME, Waller J, Tingen $\mathrm{M}$. The effect of a multi-component smoking cessation intervention in African American women residing in public housing. Res Nurs Heal. 2007;30(1):45-60. doi:10.1002/nur.20174

14. Brandon TH, Tiffany ST, Obremski KM, Baker TB. Postcessation cigarette use: The process of relapse. Addict Behav. 1990;15(2):105-114. doi:10.1016/0306-4603(90)90013-N

15. O 'hea EL, Boudreaux ED, Jeffries SK, Taylor CLC, Scarinci IC, Brantley PJ. Stage of Change Movement Across Three Health Behaviors: The Role of SelfEfficacy. Am J Heal Promot. 2004;19(2):94-102. doi:10.4278/0890-1171-19.2.94

16. Cinciripini PM, Wetter DW, Fouladi RT, et al. The effects of depressed mood on smoking cessation: Mediation by postcessation self-efficacy. J Consult Clin Psychol. 2003;71(2):292-301. doi:10.1037/0022-006X.71.2.292

17. Borland R, Yong HH, Wilson N, et al. How reactions to cigarette packet health warnings influence quitting: Findings from the ITC FourCountry survey. Addiction. 2009;104(4):669-675. doi:10.1111/j.1360-0443.2009.02508.x

18. Partos TR, Borland R, Thrasher JF, et al. The predictive utility of micro indicators of concern about smoking: Findings from the International Tobacco Control Four Country study. Addict Behav. 2014;39(8):1235-1242. doi:10.1016/j.addbeh.2014.04.001

19. Li L, Borland R, Fong GT, et al. Smoking-related thoughts and microbehaviours, and their predictive power for quitting. Tob Control. 2015;24(4):354-361. doi:10.1136/tobaccocontrol-2013-051384 
20. Cohen J. A Power Primer summary. Quant Methods Psychol. 1992;112(1):155-159. doi:10.1038/141613a0

21. Borland R, Wilson N, Fong GT, et al. Impact of graphic and text warnings on cigarette packs: Findings from four countries over five years. Tob Control. 2009;18(5):358364. doi:10.1136/tc.2008.028043

22. Li L, Borland R, Yong H, et al. Longer term impact of cigarette package warnings in Australia compared with the United Kingdom and Canada. Health Educ Res. 2015;30(1):67-80. doi:10.1093/her/cyu074

23. Evans AT, Peters E, Strasser AA, Emery LF, Sheerin KM, Romer D. Graphic warning labels elicit affective and thoughtful responses from smokers: Results of a randomized clinical trial. PLoS One. 2015;10(12). doi:10.1371/journal.pone.0142879

CONFLICTS OF INTEREST

S. G. Ferguson has consulted for GlaxoSmithKline Consumer Healthcare and Chrono Therapeutics Inc. on matters relating to smoking cessation, and has received researcherinitiated project grant funding (through the GRAND initiative) and travel funds from Pfizer. He has also served on an advisory board for Johnson \&t Johnson. D. Hammond has provided paid expert witness testimony in legal proceedings on behalf of governments, including tobacco industry challenges to health warning regulations, outside the submitted work. The rest of the authors have also completed and submitted an ICMJE form for disclosure of potential conflicts of interest. The authors declare that they have no competing interests, financial or otherwise, related to the current work.

FUNDING

There was no source of funding for this research.

PROVENANCE AND PEER REVIEW

Not commissioned; externally peer reviewed. 